\title{
DYNAMICAL CONSTRUCTION OF KÄHLER-EINSTEIN METRICS
}

\author{
HAJIME TSUJI
}

\begin{abstract}
In this article, we give a new construction of a Kähler-Einstein metric on a smooth projective variety with ample canonical bundle. As a consequence, for a dominant projective morphism $f: X \longrightarrow S$ with connected fibers such that a general fiber has an ample canonical bundle, and for a positive integer $m$, we construct a canonical singular Hermitian metric $h_{E, m}$ on $f_{*} \mathcal{O}_{X}\left(m K_{X / S}\right)$ with semipositive curvature in the sense of Nakano.
\end{abstract}

\section{$\S 1$. Introduction}

Let $X$ be a smooth projective $n$-fold with ample canonical bundle defined over $\mathbb{C}$. Then by the celebrated solution of Calabi's conjecture (see $[\mathrm{A}],[\mathrm{Y} 1]$ ), there exists a unique Kähler-Einstein $C^{\infty}$-form $\omega_{E}$ such that

$$
-\operatorname{Ric}_{\omega_{E}}=\omega_{E}
$$

holds, where Ric $\omega_{E}$ denotes the Ricci form of the Kähler manifold $\left(X, \omega_{E}\right)$.

On the other hand, for a complex manifold with very ample $L^{2}$-canonical forms, there exists a standard Kähler form called the Bergman Kähler form.

Let us explain more precisely. Let $M$ be a complex manifold of dimension $n$ such that the space of $L^{2}$-canonical forms

$$
\begin{aligned}
& H_{(2)}^{0}\left(M, \mathcal{O}_{M}\left(K_{M}\right)\right) \\
& \quad:=\left\{\eta \in H^{0}\left(M, \mathcal{O}_{M}\left(K_{M}\right)\right) \mid(\sqrt{-1})^{n^{2}} \int_{M} \eta \wedge \bar{\eta}<\infty\right\}
\end{aligned}
$$

gives a very ample linear system. Then $M$ admits a Bergman kernel,

$$
B(z, w):=\sum_{i} \sigma_{i}(z) \cdot \overline{\sigma_{i}(w)} \quad(z, w \in M)
$$

Received December 1, 2006. Revised January 29, 2009. Accepted November 26, 2009. 2000 Mathematics Subject Classification. 53C25, 32G07, 53C55, 58E11.

(C) 2010 by The Editorial Board of the Nagoya Mathematical Journal 
where $\left\{\sigma_{i}\right\}$ is a complete orthonormal basis of $H_{(2)}^{0}\left(M, \mathcal{O}_{M}\left(K_{M}\right)\right)$ with respect to the inner product

$$
\left(\eta, \eta^{\prime}\right):=(\sqrt{-1})^{n^{2}} \int_{M} \eta \wedge \bar{\eta}^{\prime} .
$$

We see that $B(z, w)$ is independent of the choice of the orthonormal basis $\left\{\sigma_{i}\right\}$ and that

$$
\omega_{B}(z):=\sqrt{-1} \partial \bar{\partial} \log B(z, z) \quad(z \in M)
$$

is a Kähler form; $\omega_{B}$ is called the Bergman Kähler form on $M$. The same construction applies for the case of the adjoint bundle of a (possibly singular) Hermitian line bundle $(L, h)$ on $M$ (see Section 3 ).

Both Kähler-Einstein metrics and Bergman metrics are determined uniquely by the complex structures. In this sense, these metrics are canonical. Hence, it is natural to study the relation of these metrics.

Recently, S. K. Donaldson [Do] found a new construction of KählerEinstein metrics or, more generally, of Kähler metrics with constant scalar curvature; actually, he found a strong connection between the existence of Kähler metrics with constant scalar curvature and the asymptotic stability of Hilbert points of projective embeddings. In particular, this implies the connection between the existence of Kähler-Einstein metrics and the asymptotic stability of Hilbert points of projective embeddings.

Let us explain a part of his results. Let $X$ be a smooth projective variety, and let $L$ be an ample line bundle on $X$. Then for every sufficiently large positive integer $m$, the linear system $|m L|$ gives a projective embedding

$$
\Phi_{m}: X \longrightarrow \mathbb{P}^{N_{m}}
$$

given by

$$
\Phi_{m}(x):=\left[\sigma_{0}^{(m)}: \cdots: \sigma_{N_{m}}^{(m)}\right]
$$

where $\left\{\sigma_{0}^{(m)}, \ldots, \sigma_{N_{m}}^{(m)}\right\}$ is a basis of $H^{0}\left(X, \mathcal{O}_{X}(m L)\right)$. Hence, $\Phi_{m}$ depends on the choice of the basis. Let $\omega_{F S}$ denote the Fubini-Study Kähler form on $\mathbb{P}^{N_{m}}$. If, for some choice of $\left\{\sigma_{0}^{(m)}, \ldots, \sigma_{N_{m}}^{(m)}\right\}$, the equality

$$
\int_{X} \frac{\sigma_{i}^{(m)} \cdot \bar{\sigma}_{j}^{(m)}}{\sum_{i=0}^{N_{m}}\left|\sigma_{i}^{(m)}\right|^{2}}\left(\Phi_{m}^{*} \omega_{F S}\right)^{n}=\delta_{i j}
$$


holds for every $0 \leqq i, j \leqq N_{m}$ (i.e., if $\left\{\sigma_{0}^{(m)}, \ldots, \sigma_{N_{m}}^{(m)}\right\}$ is orthonormal with respect to the $L^{2}$-inner product with respect to the Hermitian metric $\left(\sum_{i=0}^{N_{m}}\left|\sigma_{i}^{(m)}\right|^{2}\right)^{-2}$ on $m L$ and the volume form $\left.\left(\Phi_{m}^{*} \omega_{F S}\right)^{n}\right)$, the Kähler form

$$
\omega_{m}:=\frac{1}{m} \Phi_{m}^{*} \omega_{F S}
$$

is called balanced (or critical). The Hilbert point of $\Phi_{m}(X)$ is stable if and only if there exists a choice of the basis $\left\{\sigma_{0}^{(m)}, \ldots, \sigma_{N_{m}}^{(m)}\right\}$ such that $\Phi_{m}$ is balanced (see $[\mathrm{Z}]$ ). Donaldson's theorem is the following.

TheOrem 1.1 ([Do, page 482, Theorem 3]). Let $X$ be a smooth projective variety, and let $L$ be an ample line bundle on $X$. Suppose that $\operatorname{Aut}(X, L)$ is discrete. If $X$ admits a Kähler form $\omega$ cohomologous to $2 \pi c_{1}(L)$ with constant scalar curvature, then, for every sufficiently large $m, \Phi_{m}(X)$ is stable (i.e., $(X, L)$ is called asymptotically stable). The limit of the balanced Kähler forms $\left\{\omega_{m}\right\}$ exists in $C^{\infty}$-topology, and the limit is a Kähler form with constant scalar curvature.

In short, Theorem 1.1 gives a construction of a Kähler form with constant scalar curvature as the limit of a sequence of balanced Kähler forms, and Theorem 1.1 is closely related to the asymptotic expansion of Bergman kernels (see $[\mathrm{C}],[\mathrm{Ze}]$ ).

The purpose of this article is to construct Kähler-Einstein forms with negative Ricci curvature as a limit of Bergman Kähler forms. More precisely, the purpose of this article is to relate Kähler-Einstein forms and Bergman Kähler forms in the case of projective manifolds with ample canonical bundle.

Let us explain the construction. Let $X$ be a smooth projective $n$-fold with ample canonical bundle. Let $m_{0}$ be a positive integer such that

(1) $\left|m K_{X}\right|$ is very ample for every $m \geqq m_{0}$, and

(2) for every pseudoeffective singular Hermitian line bundle $\left(L, h_{L}\right)$ (see Definition 2.3), $\mathcal{O}_{X}\left(m_{0} K_{X}+L\right) \otimes \mathcal{I}\left(h_{L}\right)$ is globally generated.

The existence of such $m_{0}$ follows from Nadel's vanishing theorem [N, page 561].

Let $h_{m_{0}}$ be a $C^{\infty}$-Hermitian metric on $m_{0} K_{X}$ with strictly positive curvature. Suppose that we have constructed $K_{m}$ and the $C^{\infty}$-Hermitian metric $h_{m}$ on $m K_{X}$. Then we define

$$
K_{m+1}:=K\left(X,(m+1) K_{X}, h_{m}\right)
$$


and

$$
h_{m+1}:=1 / K_{m+1},
$$

where $K\left(X,(m+1) K_{X}, h_{m}\right)$ denotes the diagonal part of the Bergman kernel of $(m+1) K_{X}$ with respect to $h_{m}$ constructed as follows.

Let $\left\{\sigma_{0}^{(m+1)}, \ldots, \sigma_{N_{m+1}}^{(m+1)}\right\}$ be the complete orthonormal basis of $H^{0}(X$, $\left.\mathcal{O}_{X}\left((m+1) K_{X}\right)\right)$ with respect to the inner product

$$
(\sigma, \tau):=(\sqrt{-1})^{n_{2}} \int_{X} h_{m} \cdot \sigma \wedge \bar{\tau} \quad\left(\sigma, \tau \in H^{0}\left(X, \mathcal{O}_{X}\left((m+1) K_{X}\right)\right)\right)
$$

Then for $x \in X$ we define

$$
\begin{aligned}
K_{m+1}(x) & =K\left(X,(m+1) K_{X}, h_{m}\right)(x) \\
& :=\sum_{i=0}^{N_{m+1}}\left|\sigma_{i}^{(m+1)}\right|^{2}(x),
\end{aligned}
$$

where, for a global section $\sigma$ of $(m+1) K_{X},|\sigma|^{2}$ denotes the global section $\sigma \cdot \bar{\sigma}$ of $\left(K_{X} \otimes \overline{K_{X}}\right)^{\otimes(m+1)}$. We note that by the choice of $m_{0},\left|(m+1) K_{X}\right|$ is very ample. Hence, $h_{m+1}:=1 / K_{m+1}$ is a $C^{\infty}$-Hermitian metric on $(m+$ 1) $K_{X}$. Inductively, we construct the sequences $\left\{h_{m}\right\}_{m \geqq m_{0}}$ and $\left\{K_{m}\right\}_{m>m_{0}}$. This is the same construction originated by the author in [T3].

The following theorem is the main result in this article.

THeOREM 1.2. Let $X$ be a smooth projective $n$-fold with ample canonical bundle. Let $m_{0}$ and $\left\{h_{m}\right\}_{m>m_{0}}$ be the sequence of Hermitian metrics as above. Then

$$
h_{\infty}:=\liminf _{m \rightarrow \infty} \sqrt[m]{(m !)^{n} \cdot h_{m}}
$$

is a $C^{\infty}$-Hermitian metric on $K_{X}$ such that

$$
\omega_{\infty}:=\Theta_{h_{\infty}}
$$

is a Kähler form on $X$ with

$$
-\operatorname{Ric}_{\omega_{\infty}}=\omega_{\infty}
$$

REMARK 1.3. The existence of the limit $h_{\infty}$ has already been proved in [T3] not only for the canonically polarized varieties but also for varieties of general type (for smooth projective varieties of nongeneral type, see [T3], [T5], [T6], and [T7]). 
The construction of the Kähler-Einstein form in Theorem 1.2 is more straightforward than the one in Theorem 1.1. Also Theorem 1.2 seems to imply that the sequence of Kähler forms

$$
\left\{\frac{1}{m} \Theta_{h_{m}}\right\}_{m \geqq m_{0}}
$$

induced by the morphisms $\Phi^{(m)}: X \longrightarrow \mathbb{P}^{N_{m}}\left(m>m_{0}\right)$ defined by

$$
\Phi^{(m)}(x)=\left[\sigma_{0}^{(m)}(x): \cdots: \sigma_{N_{m}}^{(m)}(x)\right] \quad(x \in X)
$$

is asymptotically nearly balanced.

Theorem 1.2 implies the following semipositivity theorem.

THEOREM 1.4. Let $f: X \longrightarrow S$ be a projective morphism with connected fibers between smooth varieties. Let $S^{\circ}$ denote the maximal Zariski dense subset of $S$ such that $f$ is smooth over $X^{\circ}:=f^{-1}\left(S^{\circ}\right)$. Suppose that a general fiber of $f$ is a smooth projective variety with ample canonical bundle. Let $\omega_{E / S}$ be the family of relative Kähler-Einstein forms on $X^{\circ}$. Let $h_{E}^{\circ}$ be the $C^{\infty}$-Hermitian metric on $K_{X / S} \mid X^{\circ}$ defined by

$$
h_{E}^{\circ}:=\left(\omega_{E / S}^{n}\right)^{-1},
$$

where $n$ denotes the relative dimension of $f: X \longrightarrow S$. Then we have the following:

(1) $h_{E}^{\circ}$ extends to a singular Hermitian metric $h_{E}$ on $K_{X / S}$;

(2) the curvature current $\Theta_{h_{E}}$ of $h_{E}$ is semipositive on $X$;

(3) $F_{m}:=f_{*} \mathcal{O}_{X}\left(m K_{X / S}\right)$ is locally free on $S^{\circ}$, and $F_{m} \mid S^{\circ}$ carries the $C^{\infty}$-Hermitian metric $h_{E, m}$ defined by

$$
h_{E, m}(\sigma, \tau):=(\sqrt{-1})^{n^{2}} \int_{X_{s}} h_{E}^{m-1} \cdot \sigma \wedge \bar{\tau} \quad\left(\sigma, \tau \in H^{0}\left(X_{s}, \mathcal{O}_{X_{s}}\left(m K_{X_{s}}\right)\right)\right) .
$$

Then $\left(F_{m} \mid S^{\circ}, h_{E, m}\right)$ is semipositive in the sense of Nakano (see [D, VII6]).

We note that Theorems 1.2 and 1.4 can be generalized to the case of Kähler-Einstein currents (or, more generally, canonical measures; see [ST], [T7]) without any essential changes by using the existence of Kähler-Einstein currents due to Sugiyama [S] (which is a generalization of [T0]) and 
the recent result on finite generation of canonical rings (see $[\mathrm{BCHM}]){ }^{*}$ (For such further generalizations, see [T6], [T7], [T8], and [T9].)

Theorem 1.4 has several applications. For example, it immediately gives canonical positive line bundles on the moduli space of canonically polarized varieties with only canonical singularities. Such applications will be discussed in a subsequent paper ([T9]).

We should note that the convergence in Theorem 1.2 is much weaker than in Theorem 1.1. Also, Theorem 1.2 does not say anything about Kähler forms with constant scalar curvature at this moment.

\section{§2. Preliminaries}

In this section, we review the basic terminologies used in this paper.

\subsection{Singular Hermitian metrics}

In this subection, $L$ denotes a holomorphic line bundle on a complex manifold $M$.

Definition 2.1. A singular Hermitian metric $h$ on $L$ is given by

$$
h=e^{-\varphi} \cdot h_{0},
$$

where $h_{0}$ is a $C^{\infty}$-Hermitian metric on $L$ and $\varphi \in L_{\text {loc }}^{1}(M)$ is an arbitrary function on $M$. We call $\varphi$ a weight function of $h$.

The curvature current $\Theta_{h}$ of the singular Hermitian line bundle $(L, h)$ is defined by

$$
\Theta_{h}:=\Theta_{h_{0}}+\sqrt{-1} \partial \bar{\partial} \varphi
$$

where $\partial \bar{\partial}$ is taken in the sense of a current and where $\Theta_{h_{0}}=\sqrt{-1} \bar{\partial} \partial \log h_{0}$. We note that in our convention, the curvature current $\Theta_{h}$ is always a closed real current.

The $L^{2}$-sheaf $\mathcal{L}^{2}(L, h)$ of the singular Hermitian line bundle $(L, h)$ is defined by

$$
\mathcal{L}^{2}(L, h)(U):=\left\{\sigma \in \Gamma\left(U, \mathcal{O}_{M}(L)\right) \mid h(\sigma, \sigma) \in L_{\mathrm{loc}}^{1}(U)\right\},
$$

where $U$ runs over the open subsets of $M$. In this case, there exists an ideal sheaf $\mathcal{I}(h)$ such that

$$
\mathcal{L}^{2}(L, h)=\mathcal{O}_{M}(L) \otimes \mathcal{I}(h)
$$

\footnotetext{
${ }^{*}$ Actually, this paper is a part of [T6].
} 
holds. We call $\mathcal{I}(h)$ the multiplier ideal sheaf of $(L, h)$. If we write $h$ as

$$
h=e^{-\varphi} \cdot h_{0},
$$

where $h_{0}$ is a $C^{\infty}$-Hermitian metric on $L$ and where $\varphi \in L_{\text {loc }}^{1}(M)$ is the weight function, we see that

$$
\mathcal{I}(h)=\mathcal{L}^{2}\left(\mathcal{O}_{M}, e^{-\varphi}\right)
$$

holds. For $\varphi \in L_{\text {loc }}^{1}(M)$, we define the multiplier ideal sheaf of $\varphi$ by

$$
\mathcal{I}(\varphi):=\mathcal{L}^{2}\left(\mathcal{O}_{M}, e^{-\varphi}\right) .
$$

Example 2.2. Let $\sigma \in \Gamma\left(X, \mathcal{O}_{X}(L)\right)-\{0\}$ be a global section. Then

$$
h:=\frac{1}{|\sigma|^{2}}=\frac{h_{0}}{h_{0}(\sigma, \sigma)}
$$

is a singular Hermitian metric on $L$, where $h_{0}$ is an arbitrary $C^{\infty}$-Hermitian metric on $L$ (the right-hand side is obviously independent of $h_{0}$ ). The curvature $\Theta_{h}$ is given by

$$
\Theta_{h}=2 \pi(\sigma),
$$

where $(\sigma)$ denotes the current of integration over the divisor of $\sigma$.

Definition 2.3. Here $L$ is said to be pseudoeffective if there exists a singular Hermitian metric $h$ on $L$ such that the curvature current $\Theta_{h}$ is a closed positive current. Also, a singular Hermitian line bundle $(L, h)$ is said to be pseudoeffective if the curvature current $\Theta_{h}$ is a closed positive current.

\subsection{Analytic Zariski decompositions}

Let $L$ be a pseudoeffective line bundle on a compact complex manifold $X$. To analyze the ring

$$
R(X, L)=\bigoplus_{m=0}^{\infty} H^{0}\left(X, \mathcal{O}_{X}(m L)\right)
$$

it is sometimes useful to introduce the notion of analytic Zariski decompositions.

Definition 2.4. Let $M$ be a compact complex manifold, and let $L$ be a holomorphic line bundle on $M$. A singular Hermitian metric $h$ on $L$ is said to be an analytic Zariski decomposition if the following hold: 
(1) $\Theta_{h}$ is a closed positive current; and

(2) for every $m \geq 0$, the natural inclusion

$$
H^{0}\left(M, \mathcal{O}_{M}(m L) \otimes \mathcal{I}\left(h^{m}\right)\right) \rightarrow H^{0}\left(M, \mathcal{O}_{M}(m L)\right)
$$

is an isomorphism.

REMARK 2.5. If an analytic Zariski decomposition (AZD) exists on a line bundle $L$ on a smooth projective variety $M, L$ is pseudoeffective by condition (1) above.

It is known that for every pseudoeffective line bundle on a compact complex manifold, there exists an AZD on $L$ (see [T1], [T2], [DPS]).

The advantage of the AZD is that we can handle pseudoeffective line bundle $L$ on a compact complex manifold $X$ as a singular Hermitian line bundle with semipositive curvature current as long as we consider the ring $R(X, L)$.

\section{§3. Proof of Theorem 1.2}

Let $X$ be a smooth projective $n$-fold with ample canonical bundle. Let $m_{0}$ be a positive integer such that

(1) $\left|m K_{X}\right|$ is very ample for every $m \geqq m_{0}$; and

(2) for every pseudoeffective singular Hermitian line bundle $\left(L, h_{L}\right)$, $\mathcal{O}_{X}\left(m_{0} K_{X}+L\right) \otimes \mathcal{I}\left(h_{L}\right)$ is globally generated.

Let $h_{m_{0}}$ be a $C^{\infty}$-Hermitian metric on $m_{0} K_{X}$ with strictly positive curvature. Let $\left\{h_{m}\right\}_{m \geqq m_{0}}$ and $\left\{K_{m}\right\}_{m>m_{0}}$ be the sequences of Hermitian metrics and Bergman kernels constructed as in Section 1; that is, $\left\{h_{m}\right\}_{m \geqq m_{0}}$ and $\left\{K_{m}\right\}_{m>m_{0}}$ are defined inductively by

$$
K_{m+1}=K\left(X, K_{X}+m K_{X}, h_{m}\right)
$$

and

$$
h_{m+1}=1 / K_{m+1}
$$

\subsection{Upper estimate of $K_{m}$}

Let $\Delta$ be the unit open disk in $\mathbb{C}$ with center zero. Let

$$
K_{1}(\Delta):=K\left(\Delta, K_{\Delta}\right)
$$


and let $h_{1, \Delta}:=1 / K_{1}(\Delta)$. Inductively, we define $K_{m}(\Delta)(m \geqq 1)$ and $h_{m, \Delta}$ by

$$
K_{m}(\Delta):=K\left(\Delta, m K_{\Delta}, h_{m-1, \Delta}\right)
$$

and

$$
h_{m, \Delta}=1 / K_{m}(\Delta)
$$

Then by direct calculation, we see that

$$
K_{m}(\Delta)=\frac{(m+1) !}{\left(1-|z|^{2}\right)^{2 m}}\left(\frac{\sqrt{-1}}{2 \pi} d z \wedge d \bar{z}\right)^{\otimes m}
$$

holds.

Let $x \in X$, and let $\left(U, z_{1}, \ldots, z_{n}\right)$ be a local coordinate around $x$ such that $z_{1}(x)=\cdots=z_{n}(x)=0$ and $U$ is biholomorphic to the unit open polydisk $\Delta^{n}$ with center the origin via $\left(z_{1}, \ldots, z_{n}\right)$. Then by induction in $m$, it is easy to see that there exists a positive constant $C_{U}$ such that

$$
K_{m} \leqq C_{U}^{m} \cdot((m+1) !)^{n} \cdot \prod_{i=1}^{n} \frac{1}{\left(1-\left|z_{i}\right|^{2}\right)^{2 m}} \cdot\left(\bigwedge_{i=1}^{n} \frac{\sqrt{-1}}{2 \pi} d z_{i} \wedge d \bar{z}_{i}\right)^{\otimes m}
$$

holds on $U$. Hence, moving $U$, by the compactness of $X$, we have the following lemma.

Lemma 3.1. Let $d V$ be a $C^{\infty}$-volume form on $X$. Then there exists a positive constant $C_{+}$such that

$$
K_{m} \leqq C_{+}^{m} \cdot(m !)^{n} \cdot(d V)^{m}
$$

holds on $X$.

\subsection{Lower estimate of $K_{m}$}

Let $\omega_{E}$ be the Kähler-Einstein form on $X$ such that

$$
-\operatorname{Ric}_{\omega_{E}}=\omega_{E}
$$

Let $d V_{E}=(n !)^{-1} \omega_{E}^{n}$ be the volume form associated with $\left(X, \omega_{E}\right)$.

Lemma 3.2. Here

$$
\limsup _{m \rightarrow \infty} \sqrt[m]{(m !)^{-n} K_{m}} \geqq(2 \pi)^{-n} d V_{E}
$$

holds on $X$. 
Proof. Let us consider the Hermitian line bundle $\left(K_{X}, d V_{E}\right)$ on $X$. Let $p \in X$ be a point. Then by the Kähler-Einstein condition, there exists a holomorphic normal coordinate $\left(U, z_{1}, \ldots, z_{n}\right)$ such that

$$
d V_{E}^{-1}=\left\{\prod_{i=1}^{n}\left(1-\left|z_{i}\right|^{2}\right)+O\left(\|z\|^{3}\right)\right\} \cdot\left|d z_{1} \wedge \cdots \wedge d z_{n}\right|^{-2}
$$

holds. Suppose that

$$
C_{m-1} \cdot d V_{E}^{m-1} \leqq K_{m-1}
$$

holds on $X$ for some positive constant $C_{m-1}$. We note that

$$
K_{m}(x)=\sup \left\{|\sigma|^{2}(x) ; \sigma \in H^{0}\left(X, \mathcal{O}_{X}\left(m K_{X}\right)\right),(\sqrt{-1})^{n^{2}} \int_{X} h_{m-1} \cdot \sigma \wedge \bar{\sigma}=1\right\}
$$

holds for every $x \in X$, by the extremal property of the Bergman kernel. (This is well known; see, e.g., [Kr, page 46, Proposition 1.3.16].) We note that for the open unit disk $\Delta=\{t \in \mathbb{C}|| t \mid<1\}$,

$$
\int_{\Delta}\left(1-|t|^{2}\right)^{m} d t \wedge d \bar{t}=\frac{2 \pi}{m+1}
$$

holds. Then by Hörmander's $L^{2}$-estimate of $\bar{\partial}$-operator, we see that there exists a positive constant $\lambda_{m}$ such that

$$
\left(\lambda_{m} \cdot(2 \pi)^{-n} \cdot m^{n}\right) \cdot C_{m-1} \cdot d V_{E}^{m} \leqq K_{m}
$$

with

$$
\lambda_{m} \geqq 1-\frac{C}{\sqrt{m}},
$$

where $C$ is a positive constant independent of $m$.

In fact, this can be verified as follows. Let $x \in X$ be a point on $X$, and let $\left(U, z_{1}, \ldots, z_{n}\right)$ be the normal coordinate as above. We may assume that $U$ is biholomorphic to the polydisk $\Delta^{n}(r)$ of radius $r$ with center $O$ in $\mathbb{C}^{n}$ for some $r$ via $\left(z_{1}, \ldots, z_{n}\right)$.

Taking $r$ sufficiently small, we may assume that there exists a $C^{\infty}$ function $\rho$ on $X$ such that

(1) $\rho$ is identically 1 on $\Delta^{n}(r / 3)$,

(2) $0 \leqq \rho \leqq 1$, 
(3) Supp $\rho \subset \subset U$, and

(4) $|d \rho|<3 / r$, where || denotes the pointwise norm with respect to $\omega_{E}$. We note that by $(3.11)$, the mass of $\rho \cdot\left(d z_{1} \wedge \cdots \wedge d z_{n}\right)^{\otimes m}$ concentrates around the origin as $m$ tends to infinity. Hence, by (3.14) we see that the $L^{2}$-norm

$$
\left\|\rho \cdot\left(d z_{1} \wedge \cdots \wedge d z_{n}\right)^{\otimes m}\right\|
$$

of $\rho \cdot\left(d z_{1} \wedge \cdots \wedge d z_{n}\right)^{\otimes m}$ with respect to $\left(d V_{E}\right)^{-\otimes m}$ and $\omega_{E}$ is asymptotically

$$
\left\|\rho \cdot\left(d z_{1} \wedge \cdots \wedge d z_{n}\right)^{\otimes m}\right\|^{2} \sim\left(\frac{2 \pi}{m}\right)^{n}
$$

as $m$ tends to infinity, where $\sim$ means that the ratio of the both sides converges to 1 . We set

$$
\phi:=n \rho \log \sum_{i=1}^{n}\left|z_{i}\right|^{2} .
$$

We may and do assume that $m$ is sufficiently large so that

$$
m \cdot \omega_{E}+\sqrt{-1} \partial \bar{\partial} \phi>0
$$

holds on $X$.

By (3.18), the $L^{2}$-norm

$$
\left\|\bar{\partial}\left(\rho \cdot\left(d z_{1} \wedge \cdots \wedge d z_{n}\right)^{\otimes m}\right)\right\|_{\phi}
$$

of $\bar{\partial}\left(\rho \cdot\left(d z_{1} \wedge \cdots \wedge d z_{n}\right)^{\otimes m}\right)$ with respect to $e^{-\phi} \cdot\left(d V_{E}\right)^{-\otimes m}$ and $\omega_{E}$ satisfies the inequality

$$
\left\|\bar{\partial}\left(\rho \cdot\left(d z_{1} \wedge \cdots \wedge d z_{n}\right)^{\otimes m}\right)\right\|_{\phi}^{2} \leqq M_{0} \cdot\left(\frac{3}{r}\right)^{2 n+2}\left(\frac{2 \pi}{m}\right)^{n}
$$

for every $m$, where $M_{0}$ is a positive constant independent of $m$.

By Hörmander's $L^{2}$-estimate applied to the adjoint line bundle of the Hermitian line bundle $\left((m-1) K_{X}, e^{-\phi} \cdot d V_{E}^{-(m-1)}\right)$, we see that for every sufficiently large $m$, there exists a $C^{\infty}$-solution of the equation

$$
\bar{\partial} u=\bar{\partial}\left(\rho \cdot\left(d z_{1} \wedge \cdots \wedge d z_{n}\right)^{\otimes m}\right)
$$

such that

$$
u(x)=0
$$


and

$$
\|u\|_{\phi}^{2} \leqq \frac{M_{1}}{m}\left\|\bar{\partial}\left(\rho \cdot\left(d z_{1} \wedge \cdots \wedge d z_{n}\right)^{\otimes m}\right)\right\|_{\phi}^{2}
$$

hold, where \|\|$_{\phi}$ denotes the $L^{2}$-norm with respect to $e^{-\phi} \cdot d V_{E}^{-(m-1)}$ and $\omega_{E}$, respectively, and where $M_{1}$ is a positive constant independent of $m$. Then $\rho \cdot\left(d z_{1} \wedge \cdots \wedge d z_{n}\right)^{\otimes m}-u$ is a holomorphic section of $m K_{X}$ such that

$$
\left(\rho \cdot\left(d z_{1} \wedge \cdots \wedge d z_{n}\right)^{\otimes m}-u\right)(x)=\left(d z_{1} \wedge \cdots \wedge d z_{n}\right)^{m}
$$

and

$$
\left\|\rho \cdot\left(d z_{1} \wedge \cdots \wedge d z_{n}\right)^{\otimes m}-u\right\|^{2} \leqq\left(1+M_{0} \cdot\left(\frac{3}{r}\right)^{2 n+2} \sqrt{\frac{M_{1}}{m}}\right)\left(\frac{2 \pi}{m}\right)^{n}
$$

Hence, by induction on $m$, using (3.13) and (3.15), we see that there exist positive constants $C$ and $C^{\prime}$ such that, for every $m>m_{0}$,

$$
K_{m} \geqq C^{\prime}\left(\prod_{k=m_{0}}^{m}\left(1-\frac{C}{\sqrt{k}}\right)\right) \cdot(m !)^{n} \cdot(2 \pi)^{-m n} \cdot d V_{E}^{m}
$$

holds on $X$. This implies that

$$
\limsup _{m \rightarrow \infty} \sqrt[m]{(m !)^{-n} K_{m}} \geqq(2 \pi)^{-n} d V_{E}
$$

holds on $X$.

\subsection{Integral estimate of $K_{m}$}

LEMma 3.3. The inequality

$$
\int_{X} \sqrt[m]{K_{m}} \leqq\left(\prod_{k=m_{0}}^{m}\left(N_{k}+1\right)\right)^{1 / m} \cdot\left(\int_{X} \sqrt[m_{0}]{K_{m_{0}}}\right)^{m_{0} / m}
$$

holds, where $N_{k}:=\operatorname{dim}\left|k K_{X}\right|=\operatorname{dim} H^{0}\left(X, \mathcal{O}_{X}\left(k K_{X}\right)\right)-1$.

Proof. By Hölder's inequality, we have

$$
\begin{aligned}
\int_{X} \sqrt[m]{K_{m}} & =\int_{X} \frac{K_{m}^{1 / m}}{K_{m-1}^{1 /(m-1)}} \cdot K_{m-1}^{1 /(m-1)} \\
& \leqq\left(\int_{X} \frac{K_{m}}{K_{m-1}^{m /(m-1)}} \cdot K_{m-1}^{1 /(m-1)}\right)^{1 / m} \cdot\left(\int_{X} K_{m-1}^{1 /(m-1)}\right)^{(m-1) / m}
\end{aligned}
$$




$$
\begin{aligned}
& =\left(\int_{X} \frac{K_{m}}{K_{m-1}}\right)^{1 / m} \cdot\left(\int_{X} K_{m-1}^{1 /(m-1)}\right)^{(m-1) / m} \\
& =\left(N_{m}+1\right)^{1 / m} \cdot\left(\int_{X} K_{m-1}^{1 /(m-1)}\right)^{(m-1) / m} .
\end{aligned}
$$

Then continuing this process, by using

$$
\int_{X} K_{m-1}^{1 /(m-1)} \leqq\left(N_{m-1}+1\right)^{1 /(m-1)} \cdot\left(\int_{X} K_{m-2}^{1 /(m-2)}\right)^{(m-2) /(m-1)},
$$

we have that

$$
\begin{aligned}
& \int_{X}\left(K_{m}\right)^{1 / m} \\
& \quad \leqq\left\{\left(N_{m}+1\right) \cdot\left(N_{m-1}+1\right)\right\}^{1 / m} \cdot\left(\int_{X}\left(K_{m-2}\right)^{1 /(m-2)}\right)^{(m-2) / m}
\end{aligned}
$$

holds. Continuing this process, we obtain the lemma.

Using Lemma 3.3, we obtain the following.

LEMMA 3.4. The inequality

$$
\limsup _{m \rightarrow \infty} \frac{1}{(m !)^{n / m}} \int_{X}\left(K_{m}\right)^{1 / m} \leqq \frac{K_{X}^{n}}{n !}
$$

holds.

Proof. By the Kodaira vanishing theorem,

$$
H^{q}\left(X, \mathcal{O}_{X}\left(m K_{X}\right)\right)=0
$$

holds for every $m \geqq 2$ and $q \geqq 1$. Then by Hirzebruch's Riemann-Roch theorem, we have that

$$
N_{m}+1=\frac{K_{X}^{n}}{n !} m^{n}+O\left(m^{n-1}\right)
$$

holds. Then by Lemma 3.3, we have

$$
\limsup _{m \rightarrow \infty} \frac{1}{(m !)^{n / m}} \int_{X}\left(K_{m}\right)^{1 / m} \leqq \frac{K_{X}^{n}}{n !}
$$

holds. 


\subsection{Completion of the proof of Theorem $\mathbf{1 . 2}$}

By Lemma 3.1 and Lebesgue's bounded convergence theorem, we see that

$$
\limsup _{m \rightarrow \infty} \frac{1}{(m !)^{n / m}} \int_{X}\left(K_{m}\right)^{1 / m}=\int_{X}\left(\limsup _{m \rightarrow \infty} \frac{1}{(m !)^{n / m}}\left(K_{m}\right)^{1 / m}\right)
$$

holds. Hence, by Lemma 3.4, we have that

$$
\int_{X}\left(\limsup _{m \rightarrow \infty} \frac{1}{(m !)^{n / m}}\left(K_{m}\right)^{1 / m}\right) \leqq \frac{(2 \pi)^{n} K_{X}^{n}}{n !}
$$

holds. Since

$$
\int_{X} d V_{E}=\frac{1}{n !} \int_{X} \omega_{E}^{n}=\frac{(2 \pi)^{n} K_{X}^{n}}{n !}
$$

holds by the Kähler-Einstein condition, combining Lemma 3.2 and (3.38), we have the equality

$$
\limsup _{m \rightarrow \infty} \frac{1}{(m !)^{n / m}} \sqrt[m]{K_{m}}=(2 \pi)^{-n} d V_{E}
$$

This completes the proof of Theorem 1.2.

\section{§4. Proof of Theorem 1.4}

In this section, we prove Theorem 1.4 by using Theorem 1.2. Roughly speaking, Theorem 1.2 implies that what we can say about Bergman kernels also holds for Kähler-Einstein volume forms.

Proof of Theorem 1.4. Let $A$ be a sufficiently ample line bundle on $X$, and let $h_{0}$ be a $C^{\infty}$-Hermitian metric with strictly positive curvature. Then for every $s \in S^{\circ}$, we define the dynamical system of the Bergman kernels $\left\{K_{m, s}\right\}$ on the fiber $X_{s}:=f^{-1}(s)$ as in Section 1 . Then by induction on $m$, we see that the Hermitian metric

$$
h_{m} \mid X_{s}=1 / K_{m, s}
$$

on $A+m K_{X / S} \mid X^{\circ}$ has semipositive curvature by [B] or [T6, Theorem 1.4]. It also extends to a singular Hermitian metric on $A+m K_{X / S}$ with semipositive curvature by [T6, Theorem 1.4] or [BP]. Then by Theorem 1.2, we see that $h_{E}$ is a singular Hermitian metric on $K_{X / S}$ with semipositive curvature current by [T6, Theorem 1.4] or [BP]. And $h_{E}$ is smooth over $f^{-1}\left(S^{\circ}\right)$ by the well-known standard implicit function theorem argument. Then again, by [B], we see that $h_{E, m}$ defined as (1.20) has semipositive curvature in the sense of Nakano for every $m \geqq 1$. This completes the proof of Theorem 1.4. 


\section{REFERENCES}

[A] T. Aubin, Equations du type Monge-Ampère sur les varietés kähleriennes compactes, C. R. Acad. Sci. Paris Sér. A-B 283 (1976), A119-A121.

[B] B. Berndtsson, Curvature of vector bundles associated to holomorphic fibrations, arXiv:math/0511225 [math.CV]

[BP] B. Berndtsson and M. Paun, Bergman kernels and the pseudoeffectivity of relative canonical bundles, arXiv:math/0703344 [math.AG]

[BCHM] C. Birkar, P. Cascini, C. Hacon, and J. McKernan, Existence of minimal models for varieties of log general type, arXiv:math/0610203

[C] D. Catlin, "The Bergman kernel and a theorem of Tian" in Analysis and Geometry in Several Complex Variables, (Katata 1997), Trends Math., Birkhäuser, Boston, 1999, 1-23.

[D] J. P. Demailly, Complex analytic and algebraic geometry, http://www-fourier . ujf-grenoble.fr/ demailly/manuscripts/agbook.pdf (2009), 121.

[DPS] J. P. Demailly, T. Peternell, and M. Schneider, Pseudo-effective line bundles on compact Kähler manifolds, arXiv:math/0006025 [math.AG]

[Do] S. K. Donaldson, Scalar curvature and projective embeddings I, J. Differential Geom. 59 (2001), 479-522.

[Kr] S. Krantz, Function Theory of Several Complex Variables, New York, Wiley, 1982.

[N] A. M. Nadel, Multiplier ideal sheaves and existence of Kähler-Einstein metrics of positive scalar curvature, Ann. of Math. 132 (1990), 549-596.

[ST] J. Song and G. Tian, Canonical measures and Kähler-Ricci flow, arXiv:math/0802.2570

[S] K. Sugiyama, "Einstein-Kahler metrics on minimal varieties of general type and an inequality between Chern numbers" in Recent Topics in Differential and Analytic Geometry, Adv. Stud. Pure Math. 18-I, Academic Press, Boston, 1990, 417-433.

[T0] H. Tsuji, Existence and degeneration of Kahler-Einstein metrics on minimal algebraic varieties of general type, Math. Ann. 281 (1988), 123-133.

[T1] H. Tsuji, Analytic Zariski decomposition, Proc. Japan Acad. Ser. A Math. Sci. 61 (1992), 161-163.

[T2] H. Tsuji, "Existence and applications of analytic Zariski decompositions" in Analysis and Geometry in Several Complex Variables, (Katata 1997), Trends Math., Birkhäuser, Boston, 1999, 253-272.

[T3] H. Tsuji, Deformation invariance of plurigenera, Nagoya Math. J. 166 (2002), $117-134$.

[T5] H. Tsuji, Variation of Bergman kernels of adjoint line bundles, arXiv:math/0511342 [math.CV]

[T6] H. Tsuji, Dynamical construction of Kähler-Einstein metrics, arXiv:math/0606023 [math.AG]

[T7] H. Tsuji, Canonical measures and dynamical systems of Bergman kernels, arXiv:math/0805.1829

[T8] H. Tsuji, Ricci iterations and canonical Kähler-Einstein currents on log canonical pairs, arXiv:math/0903.5445

[T9] H. Tsuji, Global generation of the direct images of relative pluri log canonical systems, preprint, 2010. 
[Y1] S.-T. Yau, On the Ricci curvature of a compact Kähler manifold and the complex Monge-Ampère equation, Comm. Pure Appl. Math. 31 (1978), 339-441.

[Ze] S. Zelditch, Szögo kernel and a theorem of Tian, Int. Res. Notice 6 (1998), 317-331.

[Z] S. Zhang, Heights and reductions of semistable varieties, Compos. Math. 104 (1996), 77-105.

Department of Mathematics

Sophia University

7-1 Kioicho, Chiyoda-ku 102-8554

Japan

h-tsuji@h03.itscom.net 